\title{
Immunolocalization of markers for bone formation during guided bone regeneration in osteopenic rats
}

\author{
Tábata de Mello TERA ${ }^{1}$, Rodrigo Dias NASCIMENTO², Renata Falchete do PRADO', Mauro Pedrine SANTAMARIA ${ }^{2}$, \\ Maria Aparecida Neves JARDINI²
}

\begin{abstract}
1- Department of Bioscience and Oral Diagnosis, Instituto de Ciência e Tecnologia - ICT, Univ. Estadual Paulista - UNESP, São José dos Campos, SP, Brazil. 2- Department of Diagnosis and Surgery, Instituto de Ciência e Tecnologia - ICT, Univ. Estadual Paulista - UNESP, São José dos Campos, SP, Brazil.
\end{abstract}

Corresponding address: Maria Aparecida Neves Jardini - Department of Diagnosis and Surgery - Av. Eng. Francisco José Longo, 777 - Jd. São Dimas - São José dos Campos - 12245-000 - SP - Brazil - Phone: (55 12) 3947-9043 - Fax: (55 12) 3947-9010 - e-mail: jardini@fosjc.unesp.br

Submitted: May 12, 2014 - Modification: August 11, 2014 - Accepted: September 3, 2014

\section{ABSTRACT}

\begin{abstract}
O bjective: The aim of this paper was to evaluate the repair of onlay autogenous bone grafts covered or not covered by an expanded polytetrafluoroethylene (e-PTFE) membrane using immunohistochemistry in rats with induced estrogen deficiency. Material and Methods: Eighty female rats were randomly divided into two groups: ovariectomized (OVX) and with a simulation of the surgical procedure (SHAM). Each of these groups was again divided into groups with either placement of an autogenous bone graft alone (BG) or an autogenous bone graft associated with an e-PTFE membrane (BGM). Animals were euthanized on days $0,7,21,45$, and 60 . The specimens were subjected to immunohistochemistry for bone sialoprotein (BSP), osteonectin (ONC), and osteocalcin $(\mathrm{OCC})$. Results: All groups (OVX+BG, OVX+BMG, SHAM+BG, and SHAM+BMG) showed greater bone formation, observed between 7 and 21 days, when BSP and ONC staining were more intense. At the 45-day, the bone graft showed direct bonding to the recipient bed in all specimens. The ONC and OCC showed more expressed in granulation tissue, in the membrane groups, independently of estrogen deficiency. Conclusions: The expression of bone forming markers was not negatively influenced by estrogen deficiency. However, the markers could be influenced by the presence of the e-PTFE membrane.
\end{abstract}

Keywords: Estrogen. Bone regeneration. Osteocalcin. Osteonectin. Sialoprotein.

\section{NTRODUCTI ON}

The increased life expectancy of the population has led to increased demand for rehabilitation using osseointegrated implants and reconstructive procedures. To receive an implant, a site must have adequate alveolar bone volume $e^{1,7,13}$. When there is an inadequate alveolar ridge for this purpose, certain techniques may be used to promote an increase in bone tissue, such as autogenous bone grafts and guided bone regeneration $3,5,7,25,26,29$. Additionally, in association with the population's aging, there has been an increase in the prevalence of diseases that may interfere with the process of osseointegration, such as osteoporosis. This disease affects millions of people around the world and is characterized by decreased bone mass and structural deterioration, leading to an increased risk for fractures $9,17,25$.

Because the interference of osteoporosis with bone repair is highly debated, it is important to investigate the mechanisms by which this disease can jeopardize the osteointegration process. Several studies $7,8,10$ have aimed to describe this relationship, which observed that estrogen deficiency has a negative impact on the bone healing process during osteointegration after titanium implants were placed in OVX rats tibias, leading to less contact area between the implant surface and the bone, and showed that the bone healing of drill-hole defects in mice with OVX-induced osteoporosis exhibited impaired angiogenesis in the early stage, impaired osteogenesis in the middle stage, and impaired 
remodeling in the late stage, which resulted in compromised mechanical properties in the end.

Despite the negative effects demonstrated by some studies mentioned above, other studies have presented contradictory, and showed that ovariectomy did not seriously affect bone healing after the placement of implants in cortical bone areas, but it reduced the bone contact ratio and the bone in the cancellous bone area, and that the systemic effects of estrogen deficiency are not crucial for fracture healing. There is a lack of information about the influence of estrogen deficiency on the healing process and about the markers for bone formation after onlay autogenous bone graft placement on the mandibula.

About markers for bone formation, Langille and Solursh ${ }^{16}$ (1990) showed that culture of mesenchyme mandibular cells initiate expression of type I and II collagen at early periods, followed by type $X$, which coincided with the onset of mineralization. After that, they loste cartilage markers and began to express bone sialoprotein II (BSP II), osteocalcin, and type I collagen. In addition, osteonectin and alkaline phosphatase were demonstrable in vitro by cells in later periods. Then, the cells formed significant mineral, detected by von Kossa staining.

According to Nagata, et al. ${ }^{21}$ (1991), the initial distribution of BSP may modify bone formation and mineralization. Both osteopontin and bone sialoprotein are located ahead of the mineralization front, being necessary for the initiation of the process. Bone sialoprotein may be considered a crystal nucleator. Osteocalcin and osteonectin are not present in areas of initial crystal formation, but are seen in the entirely mineralized matrix ${ }^{27}$.

The three bone-forming markers were chosen based on their structural and mineral-inducing properties, in addition to these proteins' capacity to alter recruitment, attachment, differentiation, and activity of bone cells.

Therefore, the aim of this study was to evaluate the immunohistochemical expression of bone marker proteins in the bone repair of onlay autogenous grafts in ovariectomized rats. Additionally, the study aimed to observe whether the presence of an expanded polytetrafluoroethylene (e-PTFE) membrane has any influence on wound healing in this systemic condition.

\section{MATERI AL AND METHODS}

\section{Animals}

The present study was conducted in accordance with the Ethical Principles for Animal Experimentation adopted by the Brazilian School of Animal Experimentation (COBEA) and approved by the Research Ethics Committee at the College of
Dentistry of São J osé dos Campos - Univ. Estadual Paulista (ICT-UNESP) under protocol number 026/2008-PA/CEP.

Eighty 3-month-old, adult female Wistar rats that weighed approximately 300 grams were enrolled in the present study. During the entire period of the study, the animals were housed in groups of six in plastic cages, and food and water were given ad libitum to all the animals. Prior to the beginning of the experimental procedures, the animals were allowed to acclimatize to the laboratory environment for 5 days. The animals were randomly divided into 2 groups: Group OVX was subjected to an ovariectomy procedure, and Group SHAM was subjected to sham surgery. Each group was divided into the following two subgroups: placement of an autogenous bone graft (BG) and an autogenous bone graft associated with an e-PTFE membrane (BGM) (WL Gore, Newark, Delaware, USA).

\section{Ovariectomy}

The animals were anesthetized using a $2 \%$ xylazine solution (Rompum, Bayer, São Paulo, SP, Brazil) and ketamine (Dopalen, Agribands, Paulínia, SP, Brazil) at a ratio of $1: 1$ (0.3 ml/100 $\mathrm{g}$ of body weight). In the OVX group (40 animals), two small incisions $(\sim 10 \mathrm{~mm})$ were made beginning after the last rib, one on each side of the back of the animal. Afterward, the ovaries were held up and ligatures were made to avoid bleeding. Then, the ovaries were completely excised. In the SHAM group (40 animals), after the incisions, the bilateral ovaries were held up and then returned to their original position without being excised. The incisions were sutured using a resorbable material for the muscle layer (poligalatin 910 Vycril 4.0 - Ethicon J ohnson \& J ohnson, São J osé dos Campos, SP, Brazil) and silk sutures for the skin (4.0 Ethicon, Johnson \& Johnson, São José dos Campos, SP, Brazil)

\section{Bone graft surgery}

After 30 days, a new surgical procedure for the placement of autogenous bone grafts was performed as described by Jardini, et al. ${ }^{13}$ (2005). The calvarium was used as the donor area, and the angle of the mandible was the recipient area. The graft was initially removed using a trephine drill with an external diameter of $4.1 \mathrm{~mm}$ (Neodent, São Paulo, SP, Brazil) and then punctured in the center using a $1 / 2$ carbide bur (KG Sorensen, Cotia, $\mathrm{SP}, \mathrm{Brazil)}$ at low rotation and cooled with saline. Three perforations were made at the angle of the mandible using the $1 / 2$ carbide bur under cooling conditions, which enabled the stable attachment of the bone block to the recipient bed using a 5.0 green braided polyester suture (Ethicon, Johnson 
\& Johnson, São José dos Campos, SP, Brazil). Afterward, the e-PTFE membrane was adapted, covering the bone graft in the $O V X+B G M$ and SHAM+BGM. These procedures allowed close contact between the graft and the surface of the mandibular bone (Figure 1).

After the graft procedure, the muscle layer was sutured using a 5.0 absorbable polyglactin 910 suture (Ethicon, J ohnson \& J ohnson, São J osé dos Campos, SP, Brazil), followed by suturing of the skin using a 4.0 silk suture (Ethicon, Johnson \& Johnson, São José dos Campos, SP, Brazil). The latter was also used to suture the donor area. After the surgery, a single dose of antibiotics ( $1 \mathrm{mg} / \mathrm{kg}$ ) was administered intramuscularly to all animals (Pentabiotic, Fort Dodge, São Paulo, SP, Brazil). Euthanasia was performed using an overdose of anesthetics immediately after surgery ( 0 hour) and $7,21,45$, and 60 days after the surgical procedure (Figure 2).

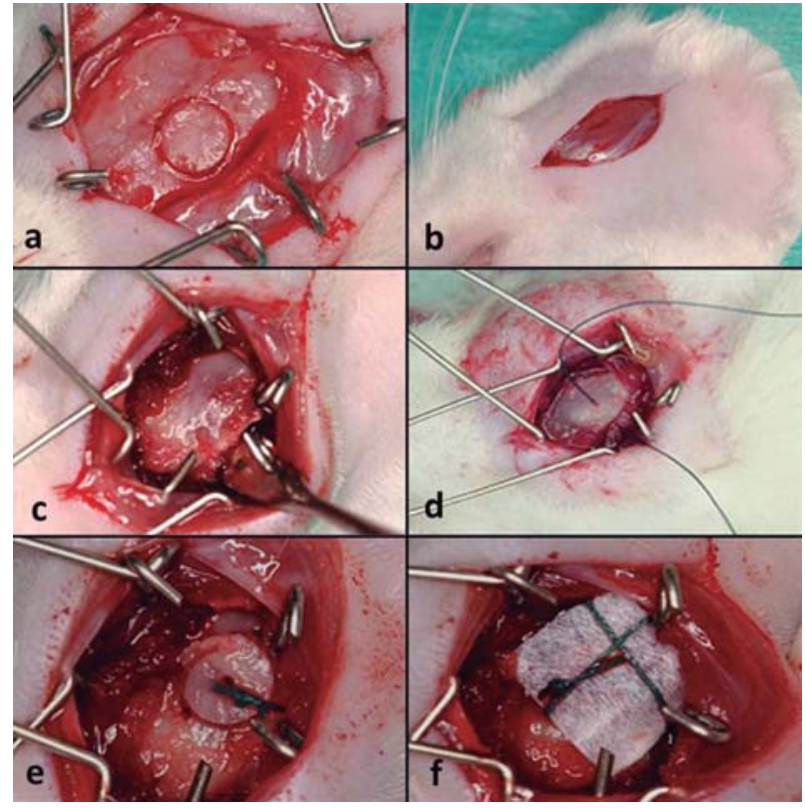

Figure 1- Surgical procedure: a) the calvarium was used as the donor area to graft removed; b) angle of the mandible was the recipient area; c) recipient bed; d) perforation was made at the angle of the mandible, which enabled the stable attachment of the bone block; e) bone block in position; f) e-PTFE membrane was adapted, covering the bone graft in the OVX+BGM and $\mathrm{SHAM+BGM}$

\section{Histological procedures}

The specimens were demineralized using a $10 \%$ EDTA solution at $\mathrm{pH} 7.8$ in a microwave oven (PELCO 3441, Ted Pella, California, USA). The demineralized specimens were sectioned transversely at the central region of the bone graft. Each fragment was placed in a paraffin block oriented toward the cutting surface.

\section{I mmunohistochemical procedures}

The blocks were sliced at a thickness of 3 $\mu \mathrm{m}$ and subjected to immunohistochemistry for osteocalcin (OCC) (FL-110: Sc-30044; Santa Cruz Biotechnology, Paso Robles, CA, USA), bone sialoprotein (BSP), and osteonectin (ONC). The BSP (LF-87) and ONC (LF-23) antibodies were kindly donated by Dr. Larry W. Fisher at the National Institute of Dental and Craniofacial Research at the National Institutes of Health (Bethesda, MD, USA).

The sections were deparaffinized in xylene. Antigen retrieval was performed using citrate $(\mathrm{pH}$ 6.0 ) in a microwave oven followed by the blocking of endogenous peroxidase using a solution of $50 \%$ methyl alcohol and hydrogen peroxide (20-volume solution) (1:1). The samples were incubated in bovine serum albumin (BSA) for 1 hour inside a moist chamber to block nonspecific antigens. Samples were then incubated with the primary antibodies (BSP, 1: 150, 1 hour, room temperature; ONC, 1:400, 1 hour, room temperature; OCC, 1:400, $4^{\circ} \mathrm{C}$, overnight) followed by incubation with a secondary antibody (Universal LSAB TM Kit/HRP, Rb/Mo/Goat - DAKO, Carpinteria, CA, USA) for 30 minutes. A final incubation was performed using the tertiary complex streptavidin peroxidase (Universal LSAB TM Kit/HRP, Rb/ Mo/Goat - DAKO, Carpinteria, CA, USA) for an additional 30 minutes. The reaction was visualized using diaminobenzidine (DAB - DAKO, Carpinteria, CA, USA). Counterstaining was performed using Mayer's hematoxylin, and the specimens were mounted in Permount.

As a positive control for BSP and ONC was used on rat bone tissue repair area. Megakaryocytes were used as a positive control for OCC. Negative controls were obtained by the omission of primary antibodies.

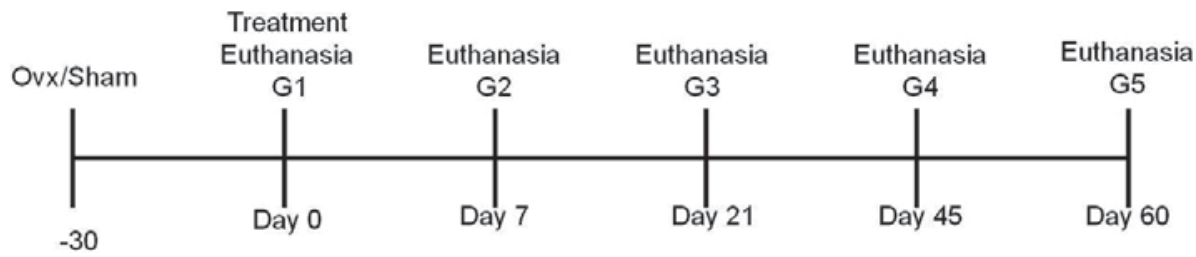

Figure 2- Periods of euthanasia 
Analysis

Microscopic analysis was conducted using an Axiophot 2 light microscope (Carl Zeiss, Oberkochen, Germany) coupled with an AxioCam MRc 5 digital camera (Carl Zeiss, Oberkochen, Germany), which transmitted images to the AxioVision Release 4.7.2 computer software.

The intensity of the immunohistochemical staining of predetermined structures and cells was classified for all periods, and antibodies were categorized as mild $(+)$, moderate $(++)$, or intense $(+++)^{12}$.

\section{RESULTS}

The OVX+BG and OVX+BGM groups presented greater marrow spaces and connective tissue compared to the SHAM groups, showing the influence of the absence of estrogen on bone repair (Figures 3, 4, and 5).

\section{BSP}

Within the same period, the 4 groups exhibited similar characteristics. At 0 hours, the bone matrix from the recipient bed and graft showed mild positivity in all groups for all periods. The OVX+BG and OVX+BMG groups showed moderate staining of the reversal lines of the recipient bed.

\begin{tabular}{|c|c|c|c|c|c|c|c|c|c|c|}
\hline & \multicolumn{2}{|c|}{ Hour 0 } & \multicolumn{2}{c|}{ Day 7 } & \multicolumn{2}{c|}{ Day 21 } & \multicolumn{2}{c|}{ Day 45 } & \multicolumn{2}{c|}{ Day 60 } \\
\hline & OVX/SHAM & OVX/SHAM & \multicolumn{2}{c|}{ OVX/SHAM } & \multicolumn{2}{|c|}{ OVX/SHAM } & \multicolumn{2}{c|}{ OVX/SHAM } \\
\hline & BG & BGM & BG & BGM & BG & BGM & BG & BGM & BG & BGM \\
\hline Bone matrix & + & + & + & + & + & + & + & + & + & + \\
\hline Newly bone RB-G & - & - & +++ & +++ & +++ & +++ & $+/++$ & $+/++$ & $+/++$ & $+/++$ \\
\hline Newly bone around G & - & - & - & - & +++ & +++ & $+/++$ & $+/++$ & $+/++$ & $+/++$ \\
\hline Osteocytes RB & $+/++$ & $+/++$ & $+/++$ & $+/++$ & $+/++$ & $+/++$ & $+/++$ & $+/++$ & $+/++$ & $+/++$ \\
\hline Osteocytes graft & ++ & ++ & ++ & ++ & ++ & ++ & ++ & ++ & ++ & ++ \\
\hline Osteocytes NB & - & - & +++ & +++ & +++ & +++ & ++ & ++ & $+/++$ & $+/++$ \\
\hline Osteoblasts & - & - & +++ & +++ & +++ & +++ & ++ & ++ & $+/++$ & $+/++$ \\
\hline Reversal lines & ++ & - & +++ & +++ & +++ & +++ & +++ & +++ & ++ & ++ \\
\hline CT B-G & - & - & - & - & - & - & - & - & - & - \\
\hline CT G & - & - & - & - & - & - & - & - & - & - \\
\hline
\end{tabular}

( $C T=$ Connective Tissue; RB=Recipient Bed; $G=$ Graft; NB= Newly Formed Bone)

$B G=$ Bone Graft; $B G M=$ Autogenous Bone Graft Associated with e-PTFE membrane

Figure 3- Bone sialoprotein (BSP). Structures labeled and staining intensity

\begin{tabular}{|c|c|c|c|c|c|c|c|c|c|c|}
\hline & \multicolumn{2}{|c|}{ Hour 0 } & \multicolumn{2}{c|}{ Day 7 } & \multicolumn{2}{c|}{ Day 21 } & \multicolumn{2}{c|}{ Day 45 } & \multicolumn{2}{c|}{ Day 60 } \\
\hline & OVX/SHAM & \multicolumn{2}{c|}{ OVX/SHAM } & \multicolumn{2}{c|}{ OVX/SHAM } & \multicolumn{2}{c|}{ OVX/SHAM } & \multicolumn{2}{c|}{ OVX/SHAM } \\
\hline & BG & BGM & BG & BGM & BG & BGM & BG & BGM & BG & BGM \\
\hline Bone matrix & - & - & - & - & - & - & - & - & - & - \\
\hline Newly bone B-G & - & - & - & - & - & - & - & - & - & - \\
\hline Newly bone around G & - & - & - & - & - & - & - & - & - & - \\
\hline Osteocytes-recipient bed & - & - & - & - & - & - & - & - & - & - \\
\hline Osteocytes graft & $+/++$ & $+/++$ & $+/++$ & $+/++$ & $+/++$ & $+/++$ & $+/++$ & $+/++$ & $+/++$ & $+/++$ \\
\hline Osteocytes NB & - & - & $+/++$ & $+/+++$ & $+/+++$ & $+/+++$ & $-/+$ & $-/+$ & - & - \\
\hline Osteoblasts & - & - & +++ & +++ & $++/+++$ & $++/+++$ & $+/+++$ & $+/+++$ & $+/++$ & $+/++$ \\
\hline Reversal lines & - & - & - & - & - & - & - & - & - & - \\
\hline CT B-G & - & - & +++ & +++ & +++ & +++ & +++ & +++ & $+/++$ & $+/++$ \\
\hline CT G & - & - & + & $++/+++$ & $+/++$ & +++ & $+/++$ & +++ & + & ++ \\
\hline
\end{tabular}

( $C T=$ Connective Tissue; RB=Recipient Bed; $G=$ Graft; NB= Newly Formed Bone)

$\mathrm{BG}=$ Bone Graft; $\mathrm{BGM}=$ Autogenous Bone Graft Associated with e-PTFE membrane

Figure 4- Osteonectin (ONC). Structures labeled and staining intensity 


\begin{tabular}{|c|c|c|c|c|c|c|c|c|c|c|}
\hline & \multicolumn{2}{|c|}{ Hour 0 } & \multicolumn{2}{c|}{ Day 7 } & \multicolumn{2}{c|}{ Day 21 } & \multicolumn{2}{c|}{ Day 45 } & \multicolumn{2}{c|}{ Day 60 } \\
\hline & OVX/SHAM & \multicolumn{2}{c|}{ OVX/SHAM } & \multicolumn{2}{c|}{ OVX/SHAM } & \multicolumn{2}{c|}{ OVX/SHAM } & \multicolumn{2}{c|}{ OVX/SHAM } \\
\hline & BG & BGM & BG & BGM & BG & BGM & BG & BGM & BG & BGM \\
\hline Bone matrix & + & + & + & + & + & + & + & + & + & + \\
\hline Newly bone RB-G & - & - & - & - & - & - & + & + & + & + \\
\hline Newly bone around G & - & - & - & - & - & - & + & + & + & + \\
\hline Osteocytes-recipient bed & + & + & + & + & + & + & + & + & + & + \\
\hline Osteocytes graft & ++ & ++ & ++ & ++ & ++ & ++ & ++ & ++ & ++ & ++ \\
\hline Osteocytes NB & - & - & + & + & & & & & & + \\
\hline Osteoblasts & - & - & $+/+$ & $+/++$ & $+/++$ & $+/++$ & +++ & +++ & +++ & +++ \\
\hline Reversal lines & - & - & - & - & - & - & - & - & - & - \\
\hline CT B-G & $+/+++$ & $+/+++$ & +++ & +++ & +++ & +++ & +++ & +++ & ++ & +++ \\
\hline CT G & - & - & $+/+++$ & +++ & $-/+$ & +++ & $-/+$ & +++ & $-/+$ & $++/+++$ \\
\hline
\end{tabular}

( $C T=$ Connective Tissue; RB=Recipient Bed; $\mathrm{G}=\mathrm{Graft}$; NB= Newly Formed Bone)

$\mathrm{BG}=$ Bone Graft; $\mathrm{BGM}=$ Autogenous Bone Graft Associated with e-PTFE membrane

Figure 5- Osteocalcin (OCC). Structures labeled and staining intensity

At 7 and 21 days, newly bone tissue formed on the surface of the recipient bed exhibited intense staining. The osteoblasts present at the periphery of the recipient bed and around the immature bone trabecular showed intense staining as well as large osteocytes, which were interspersed in the immature bone trabecular. At day 45, the newly formed bone tissue, both at the bedgraft interface and at the periphery of the graft, presented mild to moderate staining in all groups. Large osteoblasts and osteocytes in this area were moderately stained. At day 60, the newly formed bone tissue in the bed-graft interface and around the graft showed diffuse staining that ranged from mild to moderate with a predominance of mildly positive areas. Osteocytes in this area sometimes accompanied the staining, and sometimes these samples showed mild to moderate staining only in the lacuna. The osteoblasts in this region presented mild to moderate staining. The reversal lines were moderately positive (Figure $6 a$ and Figure 6b).

\section{ONC}

At 0 hours, the immunohistochemical staining was similar in all four groups. Only osteocytes located in the median region of the graft were stained, which varied from mild to moderate intensity. At day 7, the OVX+BG and SHAM+BG groups presented a large amount of intensely stained granulation tissue interposed between the graft and the recipient bed. The OVX+BGM and SHAM+BGM groups also showed this same staining pattern, though the amount of granulation tissue in this area was smaller. The granulation tissue surrounding the graft was mildly stained in the $O V X+B G$ and SHAM+BG groups, while this tissue presented moderate to intense staining in the
OVX+BGM and SHAM+BGM groups. Osteoblasts showed intense staining, and osteocytes in the immature bone trabecular exhibited varied staining that was sometimes mild and sometimes intense. The most intense staining was observed in larger osteocytes. At day 21 , the connective tissue present at the bed-graft interface presented intense staining in all four groups. The OVX+BG and SHAM+BG groups showed mild to moderate staining in the connective tissue surrounding the graft, while the OVX+BGM and SHAM+BGM groups showed intense staining. The osteoblasts showed moderate to intense staining, whereas the largest osteocytes present in the immature bone trabeculae showed more intense staining than those found in other areas. At day 45, the connective tissue interposed between the recipient bed and the graft showed intense staining in all four groups. The OVX+BG and SHAM+BG groups showed mild to moderate staining of the connective tissue surrounding the graft, whereas in the OVX+BGM and SHAM+BGM groups, the staining was intense. The osteoblasts showed mild to intense staining. The majority of the osteocytes had no staining, even the largest ones. At day 60, when present, the connective tissue at the bedgraft interface showed mild to moderate staining in all four groups. The connective tissue surrounding the graft exhibited mild staining in the BG groups and moderate staining in the $B G+M$ groups. The osteoblasts showed mild to moderate staining, while the osteocytes were not stained (Figure 7a and Figure $7 \mathrm{~b}$ ).

\section{OCC}

The bone matrix of the recipient bed and the graft showed discrete and diffuse staining in all 


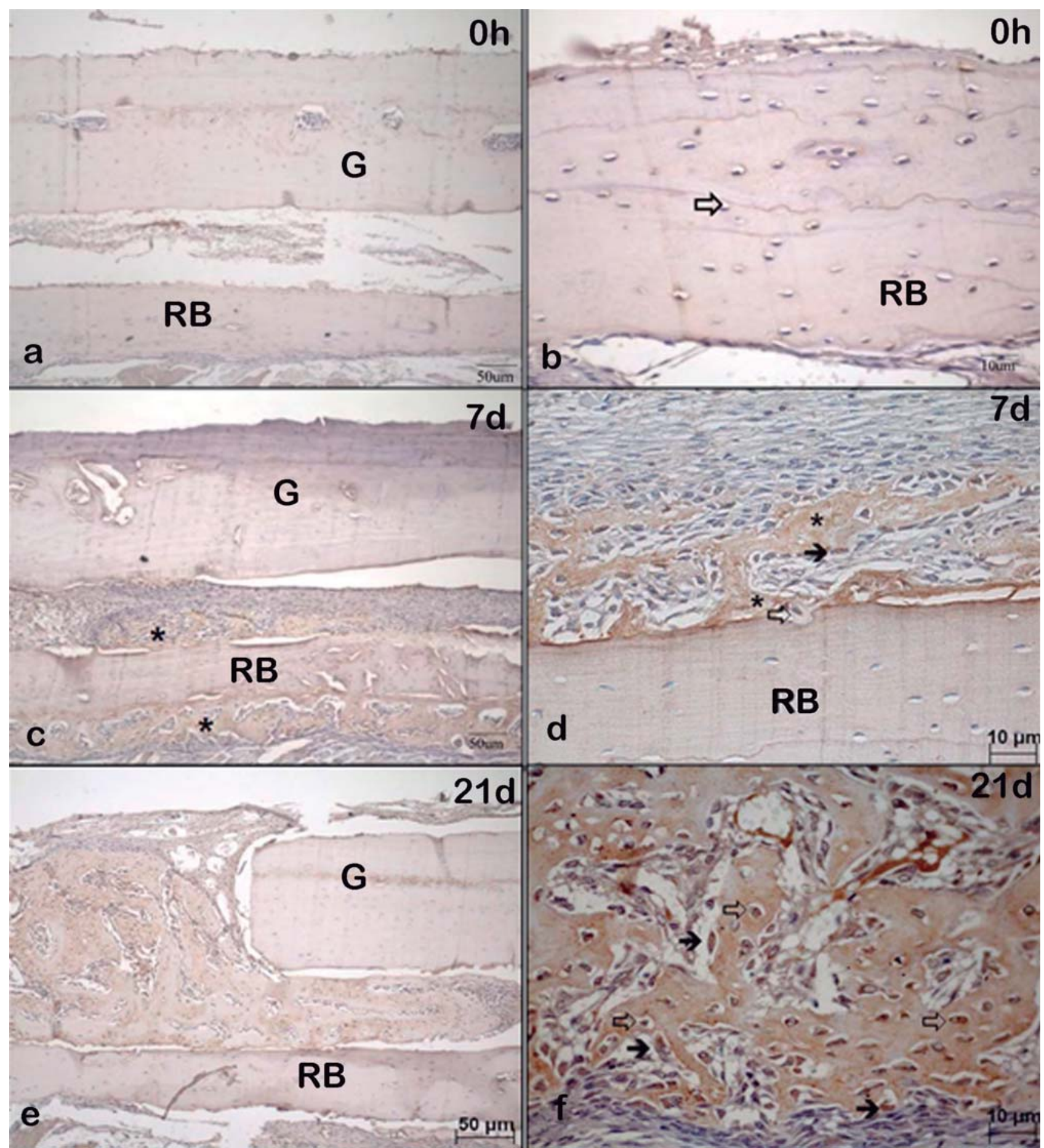

Figure 6a- a) Bone Sialoprotein (BSP) -Day 0 Group OVX-BGM: Slight expression in recipient bed (RB) matrix and graft $(G)$; b) Day 0 Group OVX-BGM: Moderate marking in reverse lines ( $\Rightarrow$ ); c) Day 7 Group SHAM-BG: Intense staining of newly formed bone $\left({ }^{*}\right)$; d) Day 7 Group SHAM-BG: Intense staining of newly formed bone $\left(^{*}\right)$, osteoblasts $(\rightarrow)$ and osteocytes; e) Day 21. Group SHAM-BGM: Intense staining of newly formed bone (*); f) Day 21 Group SHAM-BGM: Osteocytes $(\Rightarrow)$ and osteoblasts $(\rightarrow$ ) showing intense staining

evaluated groups for all periods. At 0 hours, the osteocyte lacunae present at the midline region of the graft were moderately stained, while the osteocytes of the receptor showed more discreet marking. At day 7, the newly formed bone tissue showed no staining. The OVX+BG and SHAM+BG groups exhibited large amounts of intensely stained granulation tissue interposed between the recipient bed and the graft. The OVX+BGM and SHAM+BGM groups also showed this staining pattern, though the amount of granulation tissue in this area was smaller. The granulation tissue surrounding the graft exhibited mild to intense staining in the $O V X+B G$ and SHAM+BG groups and intense 


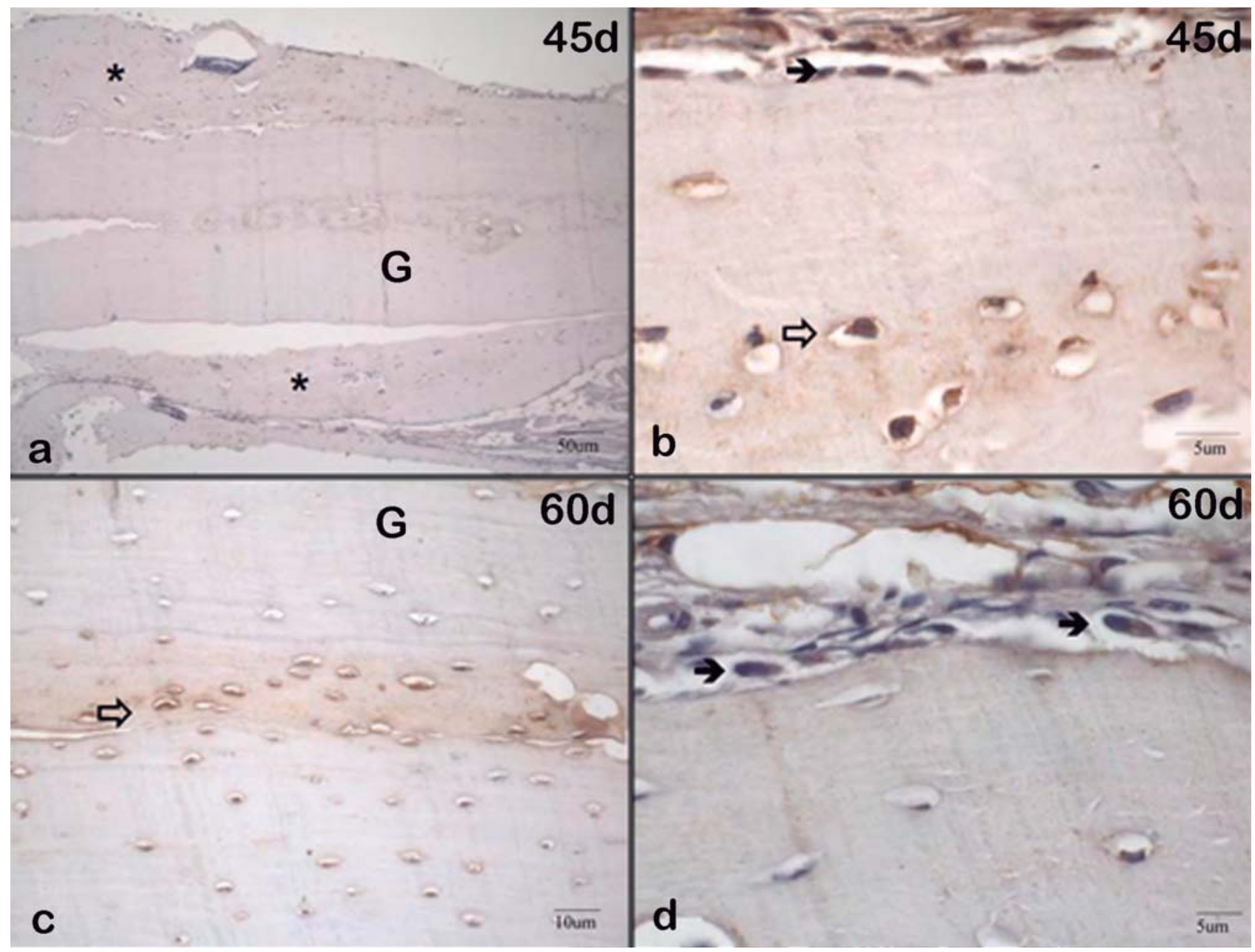

Figure 6b- a) Bone Sialoprotein (BSP) Day 45 Group SHAM-BGM: mild to moderate marking in newly formed bone ( ${ }^{*}$ ) and the middle part of the graft; b) Day 45 Group OVX-BGM: Osteoblasts $(\rightarrow)$ and osteocytes $(\Rightarrow)$ showing moderate staining; c) Day 60 Group SHAM BGM: osteocytes $(\Rightarrow)$ moderately marked on the middle portion of the graft; d) Group OVX-BGM: osteoblasts $(\rightarrow)$ showing mild to moderate staining. G: graft; RB: recipient bed

staining in the OVX+BGM and SHAM+BGM groups. The osteoblasts showed mild to moderate staining, and the large osteocytes exhibited mild staining in all four groups. At day 21, the newly formed bone tissue showed no staining. The connective tissue present at the bed-graft interface showed intense staining. In the OVX+BGM and SHAM+BGM groups, the connective tissue surrounding the graft showed intense staining, whereas, in the $O V X+B G$ and SHAM+BG groups, the staining was either negative or mild. In all groups, the osteoblasts had mild to moderate staining, and the osteocytes present in the immature bone trabecular, particularly the larger ones, showed mild staining in the SHAM+BG and SHAM+BGM groups, whereas, in the OVX+BG and OVX+BGM groups, the staining was negative. At day 45 , the newly formed bone tissue had diffuse and discrete staining at this point, similar to that observed at the initial time point. At day 60, the newly formed bone tissue in the recipient bed-graft interface exhibited mild and diffuse staining, as observed at the initial time point. This newly formed tissue was sometimes slightly more stained than the matrix. When present, connective tissues from the bed-graft interface showed intense staining in the OVX+BG and SHAM+BG groups and moderate staining in the OVX+BGM group. The connective tissue surrounding the graft showed mild or no staining in the $\mathrm{G}$ groups, whereas the staining in the BGM groups varied between moderate and intense. The osteoblasts exhibited intense staining in all regions where they were presented, like large osteocytes of the newly formed matrix (Figure $8 a$ and Figure $8 \mathrm{~b}$ ).

\section{DISCUSSION}

The aim of the present study was to evaluate the expression of immunohistochemical markers of bone formation during the repair process of autogenous bone grafts that were both covered and not covered by an e-PTFE membrane in estrogendeficient female rats. The results of the present 


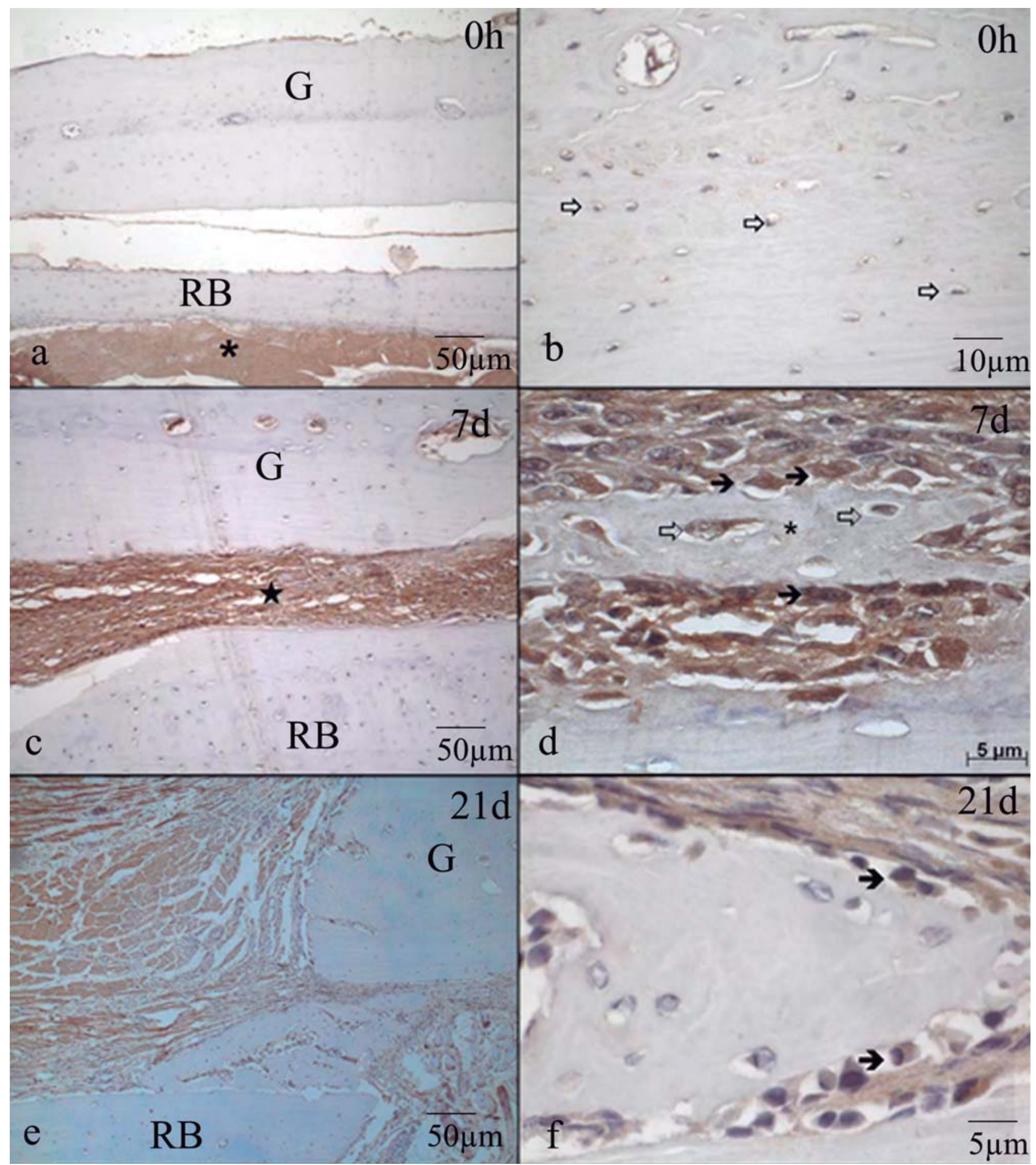

Figure 7a- a) Osteonectin (ONC) Day 0 Group OVX-BGM: no labeling of both the receptor bone matrix bed (L) and graft (E). At the bottom, intense staining was observed in skeletal striated muscle $\left(^{*}\right)$; b) Day 0 Group OVX-BGM: osteocytes $(\Longrightarrow)$ present in the mid portion of the graft exhibit mild to moderate markup; c) Day 7 Group OVX-BG: connective tissue ( $\star$ ) exhibited varied staining that was sometimes intense and sometimes mild; d) Day 7 Group SHAM-BGM: osteoblasts $(\rightarrow)$ and osteocytes $(\Longleftrightarrow)$ showing intense staining; e) Day 21 Group SHAM-BG: connective tissue present at the surround bedgraft presented intense staining; f) Day 21 Group OVX-BG: osteoblasts $(\rightarrow)$ on the bone surface are moderately marked

study demonstrate that estrogen deficiency may not alter the expression of bone markers during the repair of onlay blocks placed on rat mandibles. However, the use of the e-PTFE may enhance the expression of the bone markers regardless of the presence of the systemic condition.

The most intense staining of BSP could be observed at 7 and 21 days after surgery. The results obtained in the present study show a more intense staining of BSP in newly formed bone, 


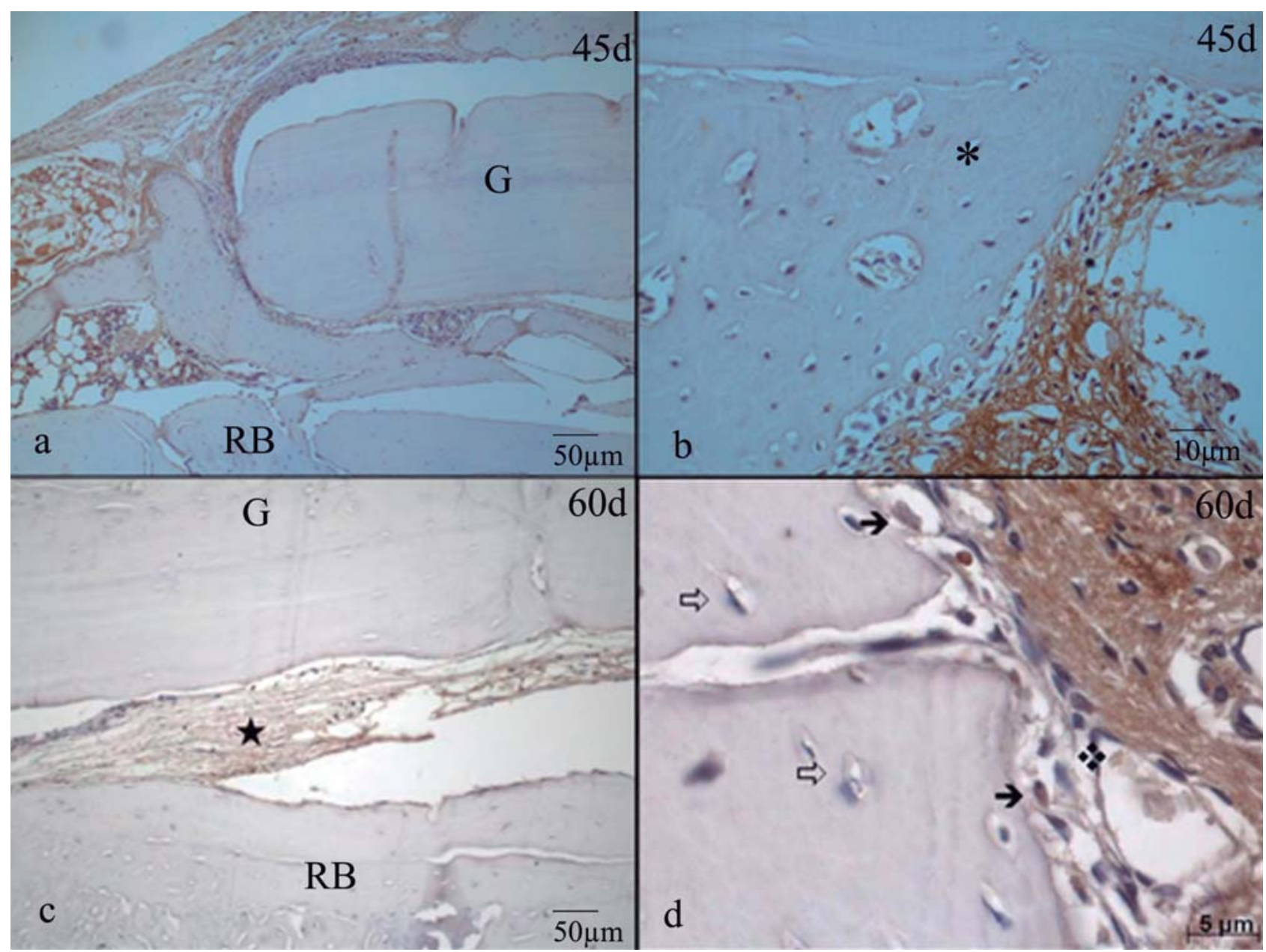

Figure 7b- a) Osteonectin (ONC) Day 45 Group OVX-BGM: in connective tissue surrounding the graft, the staining was intense; b) Day 45 Group SHAM-BGM: connective tissue surrounding newly bone ( ${ }^{*}$ ) tissue showed intense staining; c) Day 60 Group SHAM-BG: granulation tissue in graft-bed ( $\star$ ) interface lightweight markup; d) Day 60 Group OVX-BGM Group: osteoblasts $(\rightarrow)$ with mild cytoplasmic, osteocytes $(\Rightarrow)$

whereas mature bone was weakly stained at all time periods. Based on analyses of alveolar bone formation in rats, similar results were obtained by Pinero, et al. ${ }^{24}$ (1995) and Arambawatta, et al. $^{2}$ (2005). Chen, et al. ${ }^{4}$ (1993) analyzed the mandibular alveolar bone of swine fetuses and observed intense BSP staining in newly formed bone. Ivanovski et al. ${ }^{12}$ (2000) observed intense BSP staining of the newly formed bone in experiments on guided bone regeneration in dogs. In the present study, osteoblasts and osteocytes exhibited greater positivity for BSP on days 7 and 21 , which gradually decreased until the final time point, when staining was mild and restricted to the lacunae. Pinero, et al. ${ }^{24}$ (1995) observed intense BSP staining of osteoblasts in the mandibles of newborn rats, whereas the osteocytes were not stained. I vanovski, et al. ${ }^{12}$ (2000) observed intense staining of osteoblasts and osteocytes associated with newly formed bone during periodontal regeneration in dogs. Ishigaki, et al. ${ }^{11}$ (2002) observed weak BSP staining in osteoblasts present in the mandibles of fetal rats. BSP was the only marker that revealed reversal lines, and staining was slightly more pronounced in the OVX groups on days 7 and 21 .

Osteocalcin (OCC) showed staining of the newly formed bone matrix on day 45 and at 60 days, revealing characteristics of the mature bone. Similar results were found by Ivanovski, et al. ${ }^{12}$ (2000), who observed little or no staining by OCC staining of the newly formed bone in experiments on guided bone regeneration in dogs; furthermore, Ishigaki, et al. ${ }^{11}$ (2002) observed weak positivity for OCC in the newly formed bone in the mandibles of rat fetuses. Controversial results were obtained by Luvizuto, et al. ${ }^{18}$ (2010) after analyzing alveolar bone repair in ovariectomized rats. In that study, rats that were not subjected to ovariectomy showed intense staining of the newly formed bone matrix by OCC on days 14 and 21 , while the ovariectomized group showed a more discrete expression for this marker. In this study, osteoblasts showed mild to moderate staining 




Figure 8a- a) Osteocalcin (OCC) Day 0 Group OVX-BG: bone matrix with lightweight markup; b) Day 0 Group OVX-BG: moderately marked gaps of osteocytes $(\Rightarrow)$ are seen in the center of graft; c) Day 7 Group OVX-BGM: connective tissue exhibited varied staining that was sometimes intense $(\star)$; d) Day 7 Group OVZ-BGM: osteoblasts in newly bone $\left(^{*}\right)$ stained less intense than connective tissue $\left({ }^{*}\right)$; e) Day 21 OVX-BG: connective tissue in the bed-graft interface showed intense staining; f) Detail of connective tissue in Day 21 OVX-BG specimen

between 7 and 45 days, with intense staining observed at the last two time points. Osteocytes from newly formed bone presented mild positivity of their lacunae. At 21 days, staining was intense in the SHAM groups, while, in the OVX groups, it was moderate. Moderate staining was observed at 45 and 60 days. Ivanovski, et al. ${ }^{12}$ (2000) observed intense OCC staining of osteoblasts and osteocytes associated with newly formed bone during periodontal regeneration in dogs after 30 days, while I shigaki, et al. ${ }^{11}$ (2002) observed weak OCC staining in osteoblasts in the mandibles of 


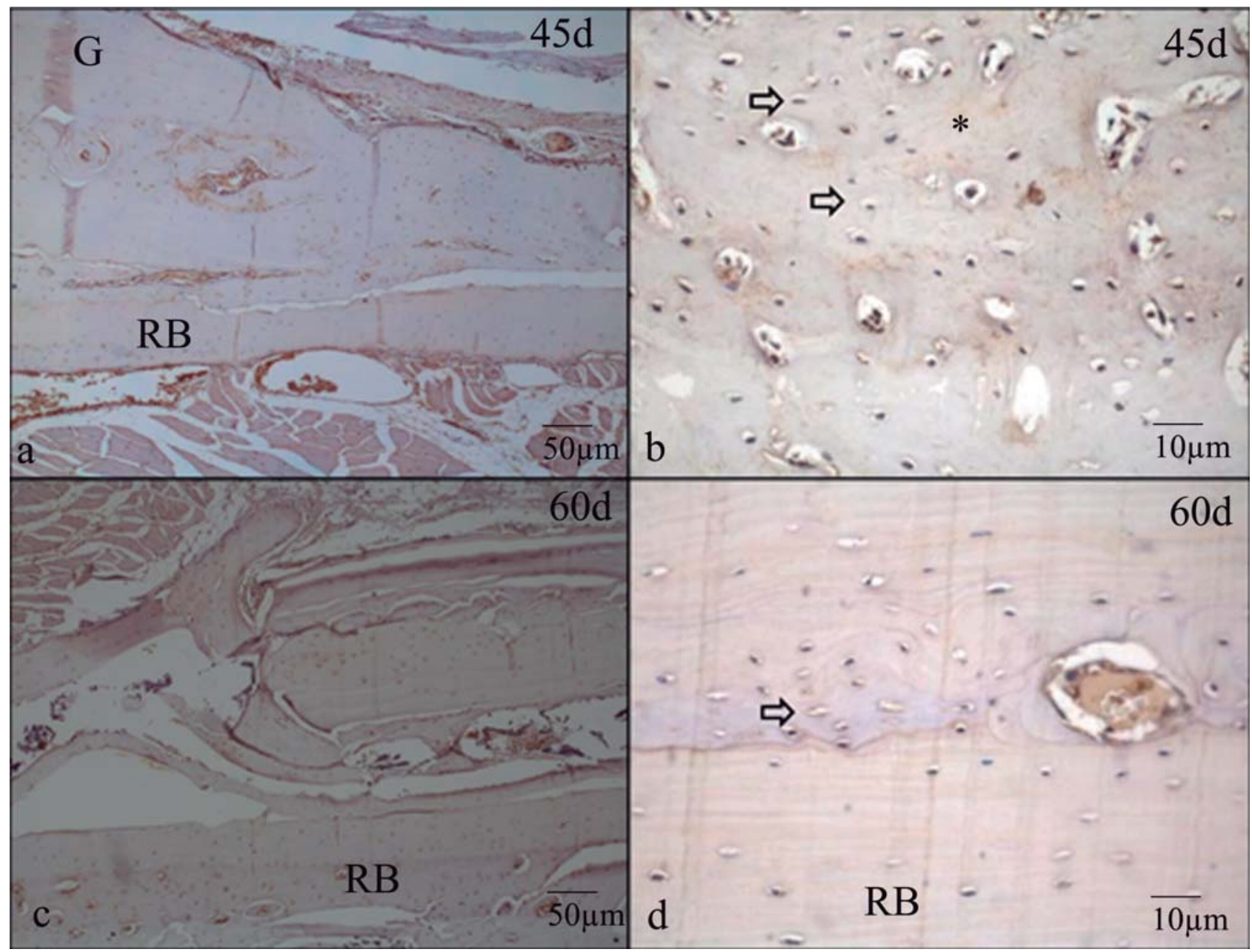

Figure 8b- a) Osteocalcin (OCC) Day 45 Group OVX-BGM: newly formed bone tissue showed diffuse and discrete staining; b) Day 45 Group OVX-BGM: in detail, newly formed bone tissue $\left(^{*}\right)$ had diffuse and discrete staining Osteocytes ( $\Rightarrow$ ); c) Day 60 Group SHAM-BGM: moderate staining in the connective tissues from the bed-graft interface; d) Day 60 Group SHAM-BG: detail of bone matrix showing discrete expression of OCC

fetal rats.

The ONC was the only marker that did not show any staining over time. Its most intense expression was observed on day 7 of the experiment. Osteoblasts showed intense positivity for ONC between 7 and 45 days. On days 7 and 21 , the ONC positivity in osteocytes from immature bone trabeculae varied from mild to intense. The most intense staining was observed in larger osteocytes. On day 45, most osteocytes were no longer stained. Similar results were found by Ishigaki, et al. ${ }^{11}$ (2002), who observed moderate to intense ONC staining in osteoblasts present in the mandibles of fetal rats.

Ovariectomy is widely recognized for its ability to induce osteopenia, as demonstrated in several classic studies ${ }^{15,20,23,25,30}$. Although several clinical studies conducted on women with osteoporosis and in animal models have demonstrated a delay


study show that ovariectomy-induced osteoporosis did not influence autogenous bone graft repair.
Melhus, et al. ${ }^{19}$ (2007) observed that estrogen deficiency was not a crucial factor in delaying bone repair.

In the present study, the results were altered by the presence of an e-PTFE membrane. Retzepi, et al. ${ }^{26}$ (2010) and Donos, et al. ${ }^{6}$ (2002) found greater bone formation, maturation, and smaller amounts of bone loss when onlay grafts were covered by e-PTFE membranes compared to grafts without the membrane. Donos, et al. ${ }^{6}$ (2002) demonstrated that the use of a membrane coating autogenous grafts accelerated the migration of osteogenic cells, the formation of new bone, and the mineralization process. Jardini, et al. ${ }^{13}$ (2005) evaluated the use of a e-PTFE membrane in specimens of autogenous grafts in rats and observed greater bone loss during the healing period in the group that did not receive the membrane. A similar experimental model developed by Nascimento, et al. ${ }^{22}$ (2009) observed that grafts not covered by the e-PTFE membrane suffered significant reabsorption.

One possible explanation for the present results 
is that estrogen deficiency may not negatively influence the expression of markers for bone formation since this condition is characterized by the increase in bone resorption and may not have any influence on bone formation. In contrast, the presence of a e-PTFE promoted an increase in intensity staining in the present study. One explanation for this may be the favorable environment for bone formation created by the presence of the barrier. According to the principle of guided bone regeneration ${ }^{7}$ there is a correlation between angiogenesis and bone regeneration.

Ridge augmentation therapy before dental implant placement is a valuable tool for oral rehabilitation. However, osteoporotic/osteopenic conditions may provide new challenges since they are a major public health threat for a large number of people around the world. Thus, it becomes necessary to recognize the impact of low bone mineral in the dental set. The results of the present study showed that the estrogen deficiency may not influence the expression of markers of bone formation. In contrast, the presence of an e-PTFE membrane created a favorable environment for bone formation. However, caution must be exercised because these results derive from an animal model, and other studies in humans and future research involving the bone forming markers of bone resorption could be useful for better understanding of the influence of estrogen deficiency on bone healing.

\section{CONCLUSION}

Within the limits of the present study, we conclude that bone metabolism during the process of bone repair was more intense between days 7 and 21 . The expression of bone forming markers may not be altered by estrogen deficiency, but the presence of an e-PTFE membrane may have a beneficial effect.

\section{ACKNOWLEDGEMENTS}

The authors gratefully acknowledge Dr. Larry W. Fisher at the National Institute of Dental and Craniofacial Research, National Institutes of Health (Bethesda, MD, USA) for kindly donating the antibodies BSP (LF-87) and ONC (LF-23), and São Paulo Research Foundation (FAPESP), which provided individual support to Maria Aparecida Neves Jardini, process no. 09/50214-1.

\section{REFERENCES}

1- Adeyemo WL, Reuther T, Bloch W, Korkmaz Y, Fisher JH, Zöller $J E$, et al. Healing of onlay mandibular bone grafts covered with collagen membrane or bovine bone substitutes: a microscopical and immunohistochemical study in the sheep. Int J Oral Maxillofac Surg. 2008; 37(7):651-9.

2- Arambawatta AKS, Yamamoto T, Wakita M. Immunohistochemical characterization of noncollagenous matrix molecules on the alveolar bone surface at the initial principal fiber attachment in rat molars. Ann Anat. 2005; 187(1):77-87.

3- Buser D, Dula K, Hirt HP, Schenk RK. Lateral ridge augmentation using autografts and barrier membranes: a clinical study with 40 partially edentulous patients. J Oral Maxillofac Surg. 1996; 54(4): 420-32

4- Chen J, McCulloch CA, Sodek J. Bone sialoprotein in developing porcine dental tissues: cellular expression and comparison of tissue localization with osteopontin and osteonectin. Arch Oral Biol. 1993; 38(3): 241-9.

5- Dahlin C, Linde A, Gottlow J, Nyman S. Healing of bone defects by guided tissue regeneration. Plast Reconstr Surg. 1988; 81(5): 672-6.

6- Donos N, Kostopoulos L, Karring T. Alveolar ridge augmentation by combining autogenous mandibular bone grafts and nonresorbable membranes. Clin Oral I mplants Res. 2002; 13(2): 18591.

7- Duarte PM, César Neto JB, Gonçalves PF, Sallum EA, Nociti FH. Estrogen deficiency affects bone healing around titanium implants: a histometric study in rats. Implant Dent. 2003; 12(4): 340-6.

8- Duarte PM, Gonçalves P, Casati MZ, Toledo S, Sallum EA, Nociti FH J r. Estrogen and alendronate therapies may prevent the influence of estrogen deficiency on the tooth-supporting alveolar bone: a histometric study in rats. J Periodontal Res. 2006; 41(6): 541-6.

9- Hao YJ, Zhang G, Wang YS, Qin L, Hung WY, Leung K, et al. Changes of microstructure and mineralized tissue in the middle and late phase of osteoporotic fracture healing in rats. 2007; 41(4): 631-8.

10- He YX, Zhang G, Pan XH, Liu Z, Zheng LZ, Chan CW, et al. I mpaired bone healing pattern in mice with ovariectomy-induced osteoporosis: a drill-hole defect model. 2011;48(6) : 1388-400. 11- Ishigaki R, Takagi M, Igarashi M, Ito K. Gene expression and immunohistochemical localization of osteonectin in association with early bone formation in the developing mandible. Histochem J. 2002;34(1-2):57-66.

12- I vanovski S, Li H, Daley T, Bartold PM. An immunohistochemical study of matrix molecules associated with barrier membranemediated periodontal wound healing. J Periodontal Res. 2000; 35(3): 115-26.

13- Jardini MA, De Marco AC, Lima LA. Early healing pattern of autogenous bone grafts with and without e-PTFE membranes: a histomorphometric study in rats. Oral Surg Oral Med Oral Pathol Oral Radiol Endod. 2005; 100(6):666-73.

14- Jardini MA, Marco AC, Melo Filho AB, Nascimento RD, Kerbauy WD, Santamaria MP. Analysis of the volume of autogenous cancellous bone grafts with or without the use of ePTFE membranes in ovariectomized rats. Braz Dent Sci. 2013; 16(3): 35-46.

15- Kalu DN. The ovariectomized rat model of postmenopausal bone loss. Bone Miner. 1991; 15(3): 175-91.

16- Langille RM, Solursh M. Formation of chondrous and osseous tissues in micromass cultures of rat frontonasal and mandibular ectomesenchyme. Differentiation. 1990;44(3): 197-206.

17- Lerner UH. Bone remodeling in post-menopausal osteoporosis. J Dent Res. 2006; 85(7): 584-95.

18- Luvizuto ER, Dias SM, Queiroz TP, Okamoto T, Garcia Junior $I R$, Okamoto $R$, et al. Osteocalcin immunolabeling during the alveolar healing process in ovariectomized rats treated with estrogen or raloxifene. Bone. 2010;46(4): 1021-9. 
19- Melhus G, Solberg LB, Dimmen S, Madsen JE, Nordsletten L, Reinholt FP. Experimental osteoporosis induced by ovariectomy and vitamin D deficiency does not markedly affect fracture healing in rats. Acta Orthop. 2007;78(3):393-403.

20- Motohashi M, Shirota T, Tokugawa Y, Ohno K, Michi K, Yamaguchi A. Bone reactions around hydroxyapatite-coated implants in ovariectomized rats. Oral Surg Oral Med Oral Pathol Oral Radiol Endod. 1999; 87(2): 145-52.

21- Nagata T, Goldberg HA, Zhang Q, Domenicucci C, Sodek J. Biosynthesis of bone proteins by fetal porcine calvariae in vitro. Rapid association of sulfated sialoproteins (secreted phosphoprotein-1 and bone sialoprotein) and chondroitin sulfate proteoglycan (CS-PGIII) with bone mineral. Matrix. $1991 ; 11(2): 86-100$.

22- Nascimento RD, Cardoso PE, De Marco AC, Lima LA, Jardin MAN. Influence of osteopenia in autogenous bone graft healing with or without expanded polytetrafluoethylene membranes: histologic and histomorphometric study in rats. Int J Oral Maxillofac Implants. 2009;24(6): 1074-82.

23- Pan J, Shirota T, Ohno K, Michi K. Effect of ovariectomy on bone remodeling adjacent to hydroxyapatite-coated implants in the tibia of mature rats. J Oral Maxillofac Surg. 2000; 58(8):87782.
24- Pinero GJ, Farach-Carson MC, Devoll RE, Aubin J E, Brunn J C, Butler WT. Bone matrix proteins in osteogenesis and remodelling in the neonatal rat mandible as studied by immunolocalization of osteopontin, bone sialoprotein, alpha 2HS-glycoprotein and alkaline phosphatase. Arch Oral Biol. 1995; 40(2): 145-55.

25- Rachner TD, Khosla S, Hofbauer LC. Osteoporosis: now and the future. Lancet. 2011;377(9773): 1276-87.

26- Retzepi M, Donos N. Guided bone regeneration: biological principle and therapeutic applications. Clin Oral Implants Res. 2010; 21(6): 567-76.

27- Roach HI. Why does bone matrix contain non-collagenous proteins? The possible roles of osteocalcin, osteonectin, osteopontin and bone sialoprotein in bone mineralisation and resorption. Cell Biol Int. 1994; 18(6):617-28.

28- Shoji K, Elsubeihi ES, Heersche J N. Effects of ovariectomy on turnover of alveolar bone in the healed extraction socket in rat edentulous mandible. Arch Oral Biol. 2011;56(2):114-20 29- Taguchi $Y$, Amizuka N, Nakadate M, Ohnishi H, Fujii $\mathrm{N}$, Oda $\mathrm{K}$, et al. A histological evaluation for guided bone regeneration induced by a collagenous membrane. Biomaterials. 2005; 26(31): 6158-66.

30- Wronski TJ, Cintrón M, Dann LM. Temporal relationship between bone loss and increased bone turnover in ovariectomized rats. Calcif Tissue Int. 1988;43(3):179-83. 